\title{
Lymph node status in different molecular subtype of breast cancer: triple negative tumours are more likely lymph node negative
}

\author{
Ning Liu ${ }^{1}$, Zhigang Yang ${ }^{2}$, Xiaozhen Liu ${ }^{3}$ and Yun Niu ${ }^{3}$ \\ ${ }^{1}$ Department of Pathology, Bao Di Hospital, Bao Di Clinical College of Tianjin Medical University, Tianjin, China \\ 2 Tianjin Baodi Hospital of Traditional Chinese Medicine, Tianjin, China \\ ${ }^{3}$ Tianjin Medical University Cancer Institute and Hospital, National Clinical Research Center for Cancer, Key Laboratory \\ of Cancer Prevention and Therapy of Tianjin, Key Laboratory of Breast Cancer Prevention and Therapy, Tianjin Medical \\ University, Ministry of Education, Tianjin, China
}

Correspondence to: Ning Liu, email: liunings2016@126.com

Keywords: breast cancer, molecular subtype, triple negative subtype, lymph node status, prognosis

Received: October 25, $2016 \quad$ Accepted: January 09, $2017 \quad$ Published: February 02, 2017

Copyright: Liu et al. This is an open-access article distributed under the terms of the Creative Commons Attribution License 3.0 (CC BY 3.0), which permits unrestricted use, distribution, and reproduction in any medium, provided the original author and source are credited.

\section{ABSTRACT}

Background and Objectives: To investigate the association between different molecular subtype (MST) and the axillary lymph nodal (ALN) status.

Materials and Methods: A total of 528 female patients with primary breast cancer were collected. Survival estimates were calculated using the Kaplan-Meier method, univariate and multivariate logistic regression models.

Results: Triple negative and Luminal A breast cancers were more frequently node-negative (NO) when compared to Luminal B and Her-2 positive cancers $\mathbf{7 7 . 4 \%}$ and $73.4 \%$ vs. $45.3 \%$ and $40.0 \%$, respectively; $P<0.0001$ ). We observed a clearly significant difference among ALN status in patients with Her-2 positive $(P=0.001)$ and Luminal $B(P<0.0001)$ breast cancer. While no significant prognostic diffreence among different $L N$ status was detected in the Triple negative $(P=0.070)$ and Luminal A subtype $(P=0.660)$. On the other hand, we detected no prognostic diffreence among different MST in $\mathrm{N} 1$ and $\mathrm{N} 3$ subgroups $(P=0.569$ and $P=0.484$, respectively). Multivariate analysis showed that lymph node status $(P<0.01)$, molecular subtype ( $P$ $<0.01)$, and tumor size $(P<0.01)$ were significantly and independently prognostic factors. The c-index of the prognosis nomogram for recurrence prediction was 0.70 .

Conclusion: Triple negative breast cancer is not associated more frequently with a higher number of involved nodes. The prognosis nomogram can predict the probability of recurrence patients within 3 or 5 years.

\section{INTRODUCTION}

Axillary lymph nodal (ALN) status is one of the most robust factors correlated to overall survival in breast cancer patients, and as such, it has been an integral component of the staging, prognosis, and treatment of invasive breast cancer [1-3]. Recently, it is well described that breast cancer molecular subtype (MST) are associated with significant differences in prognosis [4-5]. Although several predictors(such as multifocality, higher tumor grade, larger tumor size, and the presence of lymphovascular invasion) of lymph node metastasis have been described $[1,6]$, the impact of tumor MST on ALN has not been well established [4, 7-8].

Breast cancer composed of at least four major subtypes, namely Luminal A, Luminal B , Her-2 positive and Triple negative breast cancer [4]. Triple negative breast cancer exhibits more aggressive clinical behavior, higher metastatic potential, and poorer prognosis compared to other subtypes, and characterized by an adverse prognosis particularly in case of limited sensitivity against neoadjuvant chemotherapy [9-11]. Despite their aggressive clinical behavior, some studies have shown that lymph node involvement may be less frequent in the Triple negative subtype of breast cancer [12-14]. 
The aim of this study was to confirm the lower risk of ALN involvement at the time of diagnosis in Triple negative breast cancer patients. In addition, we investigated the association between different molecular subtype and the ALN status in Chinese women diagnosed with primary breast cancer between 2004 and 2009 .

\section{MATERIALS AND METHODS}

\section{Patient selection}

We identified 528 cases femal patients with invasive breast cancer diagnosed at two clinical research center (212 cases from Bao Di Clinical College of Tianjin Medical University and 316 cases from Cancer Hospital of Tianjin Medical University, China) from Jan 1, 2004 to Dec 31, 2009. The enrolled patients met the following criterion: (1) breast cancer as the first and only cancer diagnosis, (2) equal to or greater than 10 lymph nodes dissected to ensure adequate nodal clearance, (3) known lymph node size, (4) known ER, PR, Her-2, Ki67, p53 information, (5) no previous neoadjuvant systemic therapy, (6) completed follow-up date during the study period. The exclusion criteria were as following: (1) patients with ductal carcinoma in situ(DCIS), (2) patients who received breast conserving surgery, (3) recurrent breast cancer, (4) metastatic breast cancer, (5) patients who underwent neoadjuvant systemic therapy, (6) no sufficient data to allow for the estimation of a hazard ratio (HR) with $95 \%$ confidence intervals $(95 \% \mathrm{CI})$.

Lymph node (LN) status was then evaluated based on number of tumor involved axillary lymph node (ALN). A positive node was defined as a lymph node containing any cancer cells by hematoxylin and eosin stain or cytokeratin positivity via immunohistochemistry (IHC). Women with micrometastases or macrometastases in the ALNs were considered LN positive. Women with only isolated tumor cells (ITCs) in the ALNs were considered LN negative. In the study period, micrometastases were defined as metastases between 0.2 and $2 \mathrm{~mm}$ or a tumor cell count between 10 and 100 . In the same period, ALN metastases smaller than $0.2 \mathrm{~mm}$ and with a tumor cell count less than 10 were defined as ITCs [15]. According to the above criterion, $\mathrm{LN}$ status was divided into four groups: 0 node positive (N0), 1-3 nodes positive (N1), 4-9 nodes positive (N2), more than or equal to 10 nodes positive (N3).

\section{Immunohistochemical evaluation}

The status of estrogen receptor (ER), progesterone receptor (PR), Her-2, Ki67, and p53 was determined by immunohistochemistry (IHC) and collected from pathology reports. IHC was performed using standard procedures. ER and PR were categorized as negative $(<1 \%)$ and positive $(\geq 1 \%)$ of tumor cell nuclear staining, in accordance with recent guidelines [16]. Her-2 was scored for the intensity and the completeness of cell membrane staining based on the 2013 ASCO/CAP guidelines (-, no staining; +, weak partial membranous staining in more than $10 \%$ tumor cells; ++ , moderately complete membrane staining in more than or equal to 10 $\%$ tumor cells or strong complete membranous staining in less than or equal to $10 \%$ of tumor cells; +++ , strong complete membranous staining in more than $10 \%$ of tumor cells). Her-2 (+++) was defined as positive. FISH assay was performed in selected cases (i.e., those with ++ immunoreactivity) [17]. Ki67 status was expressed in terms of percentage of positive cells, with a threshold of $20 \%$ of positive cells [18]. For p53, positive staining of fewer than $10 \%$ of the tumor cells was defined as negative expression and staining of $10 \%$ or more of the tumor cells as positive expression [19-20]. Because the lymphatic vessels and small veins is difficult to identify, lymphatic vessels and fine veins are collectively tend to referred to as lymphovascular under routine pathological. Lymphatic cancer struck and fine veins around the tumor showed lymphovascular invasion positive .

Based on 2013 St. Gallen Consensus, subtypes of breast cancer (Luminal A, Luminal B, Her-2 positive, and Triple negative) were defined by ER, PR, Ki67, and Her-2 status [18]: Luminal A (ER+ and PR $\geq 20 \%$, Her-2-, Ki67 $<20 \%$ ); Luminal B which include Luminal B-Her2-negative-like (ER+ and PR-/ $<20 \%$, Her-2-, Ki67 $\geq 20$ $\%$ ), and Luminal B-Her-2-positive-like (ER+ and Her-2+, any PR and Ki67); Her-2 positive (nonluminal: Her-2+, ER- and PR-); Triple negative (basal-like: Her-2-, ER- and PR -).

\section{Follow-up study and study endpoints}

Follow-up data were obtained via medical records, making telephone calls and study questionnaire. The primary endpoints were recurrence-free survival (RFS). RFS was calculated as time from surgery to locoregional recurrence (tumor recurrence in the ipsilateral chest wall, axilla, and infraclavicular, supraclavicular, or internal mammary lymph nodes), distant metastasis, or death. The last follow-up date was defined as the last breast cancer evaluation by a physician or a mammogram.

\section{Statistics}

The chi-square test was used to evaluate the relationship between the clinicopathologic variables and the lymph nodes status. The Kaplan-Meier method were used for the RFS analyses. Univariate analyses were performed using Cox proportional hazard models. Multivariate analyses were performed on features 
Table 1: Clinicopathologic characteristics among different lymph node status

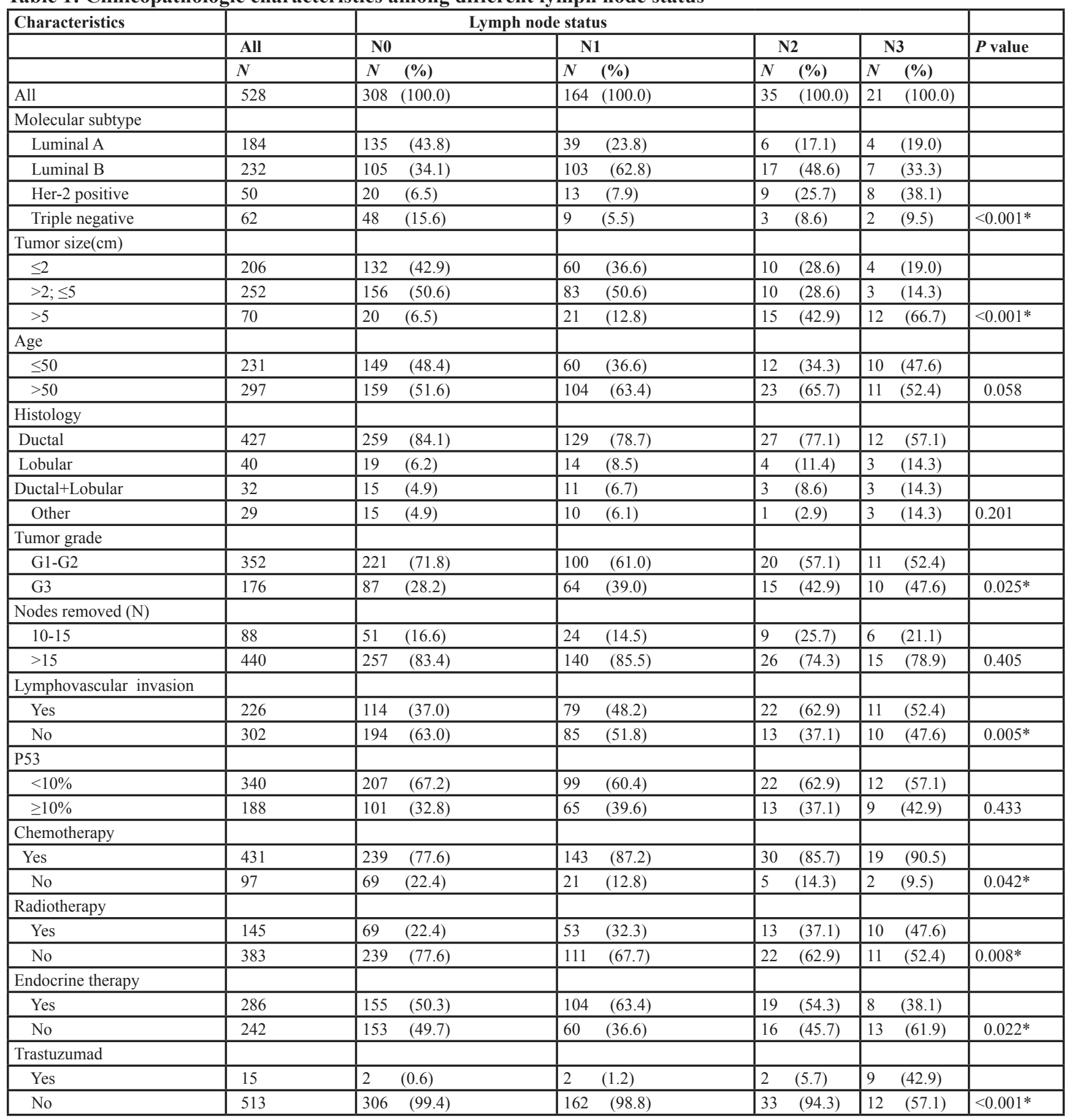

*Difference was statistically significant

including significant clinical and biological features at univariate analysis. Multivariable logistic regression was used to estimate the association of number of positive lymph nodes with a number of variables. Hazard ratio (HR), 95\% confidence intervals (CI) and $P$-values were all calculated. All $P$-values were two-sided, and $P$-values $<0.05$ were considered to be statistically significant. All analyses were conducted using IBM Statistics SPSS 19.0.

\section{Nomogram development}

The Cox proportional hazards regression model was used to construct the nomogram. The model performance was quantified with respect to discrimination and calibration. Discrimination (i.e., whether the relative ranking of individual predictions is in the correct order) was quantified using the concordance index (c-index). The c-index ranges from 0 to 1 , with 1 indicating perfect 
Table 2: Multivariable analysis of predictors of lymph node positivity

\begin{tabular}{|l|c|c|c|}
\hline \multicolumn{1}{|c|}{ Variable } & HR & $\mathbf{9 5 \% C I}$ & P-value \\
\hline Age $(>50$ vs. $\leq 50)$ & 1.59 & $1.03-2.46$ & $0.039^{*}$ \\
\hline Tumor grade (G3 vs. G1-G2) & 1.14 & $0.86-1.53$ & 0.362 \\
\hline LVI (Yes vs. No) & 1.47 & $1.10-1.95$ & $0.010^{*}$ \\
\hline Tumor size ( $>$ 2cm; $\leq$ 5cm vs. $\leq 2 \mathrm{~cm})$ & 0.92 & $0.66-1.28$ & 0.604 \\
\hline ( $>$ 5cm vs. $\leq 2 \mathrm{~cm})$ & 1.62 & $1.07-2.44$ & $0.022^{*}$ \\
\hline MST ( Luminal B vs. Luminal A) & 1.52 & $1.04-2.22$ & $0.032^{*}$ \\
\hline (Her-2 positive vs. Luminal A) & 2.30 & $1.21-4.38$ & $0.011^{*}$ \\
\hline (Triple negative vs. Luminal A) & 0.75 & $0.41-1.40$ & 0.763 \\
\hline (Triple negative vs. Her-2 positive) & 0.88 & $0.59-1.32$ & 0.687 \\
\hline
\end{tabular}

LVI: Lymphovascular invasion; MST: Molecular subtype

*Difference was statistically significant

concordance, 0.5 indicating no better concordance than chance, and 0 indicating perfect discordance [19-20].

\section{RESULTS}

\section{Patient characteristics}

We included in the analysis 528 women with invasive breast cancer treated between Jan 1, 2004 to Dec 31,2009 . The characteristics of the evaluable patients by lymph node status are given in Table 1 . The age of the patients ranged from 18 to 85 years with a mean age and median age of 52.1 years and 51 years, respectively. The N0 consisted of 308 patients $(58.3 \%)$, the N1 consisted of 164 patients $(31.1 \%)$, the N2 consisted of 35 patients $(6.6 \%)$, and the N3 consisted of 21 patients $(4.0 \%)$. The breakdown by molecular subtype included 184 (34.8\%) patients with Luminal A, 232 (43.9\%) with Luminal B, $50(9.5 \%)$ with Her-2 positive, and $62(11.8 \%)$ with Triple negative. Patients with no LN metastasis (N0) had more Triple negative tumors compared to N1, N2, N3 patitnts (15.6\% vs. 5.5\%, 8.6\%, and 9.5\%; $P<0.0001)$. Patients with N0 had more Luminal A tumors compared to N1, N2,
N3 patitnts (43.8\% vs. 23.8\%, 17.1\%, and 19.0\%). Patients with N3 were more likely to have larger tumor size ( $>$ $5 \mathrm{~cm} ; P<0.001)$, higher tumor grade $(\mathrm{G} 3 ; P=0.025)$ when compared to N0, N1, N2 patitnts. Patients with N2 were more likely to have lymphovascular invasion (LVI) $(P=0.005)$. Regarding to treatment, patients with N3 were more likely to received chemotherapy and radiotherapy. Triple negative tumours are more likely lymph
node negative

As shown in Figure 1, Triple negative and Luminal A breast cancers were more frequently node-negative (N0) when compared to Luminal B and Her-2 positive cancers (77.4\% and $73.4 \%$ vs. $45.3 \%$ and $40.0 \%$, respectively; $P$ $<0.001)$. On univariate analysis, data suggest that breast cancer subtypes are predictive of lymph node positivity. On multivariable analysis, when adjusted for tumor size, tumor grade, age, and presence of LVI, predictors of LN positivity included Age $>50$ (odds ratio [OR] 1.59, 1.032.46), presence of LVI (OR 1.47; 1.10-1.95), and tumor size $>5 \mathrm{~cm}(\mathrm{OR} 1.62 ; 1.07-2.44)$. When compared to the Luminal A subtype, the odds ratio for $\mathrm{LN}$ positivity in Triple negative was 0.75 , with $95 \%$ CI of $0.41-1.40$,

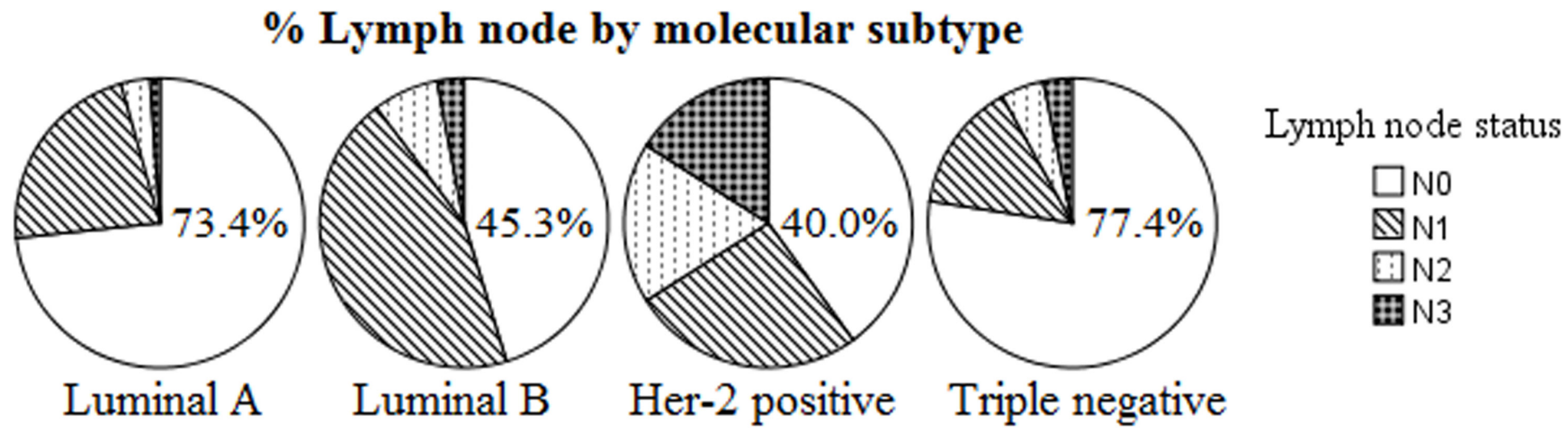

Figure 1: Number of positive lymph nodes by molecular subtype $(P<\mathbf{0 . 0 0 0 1})$. More N0 in Luminal A and Triple negative, more N2 in Her-2 positive and Luminal B. 

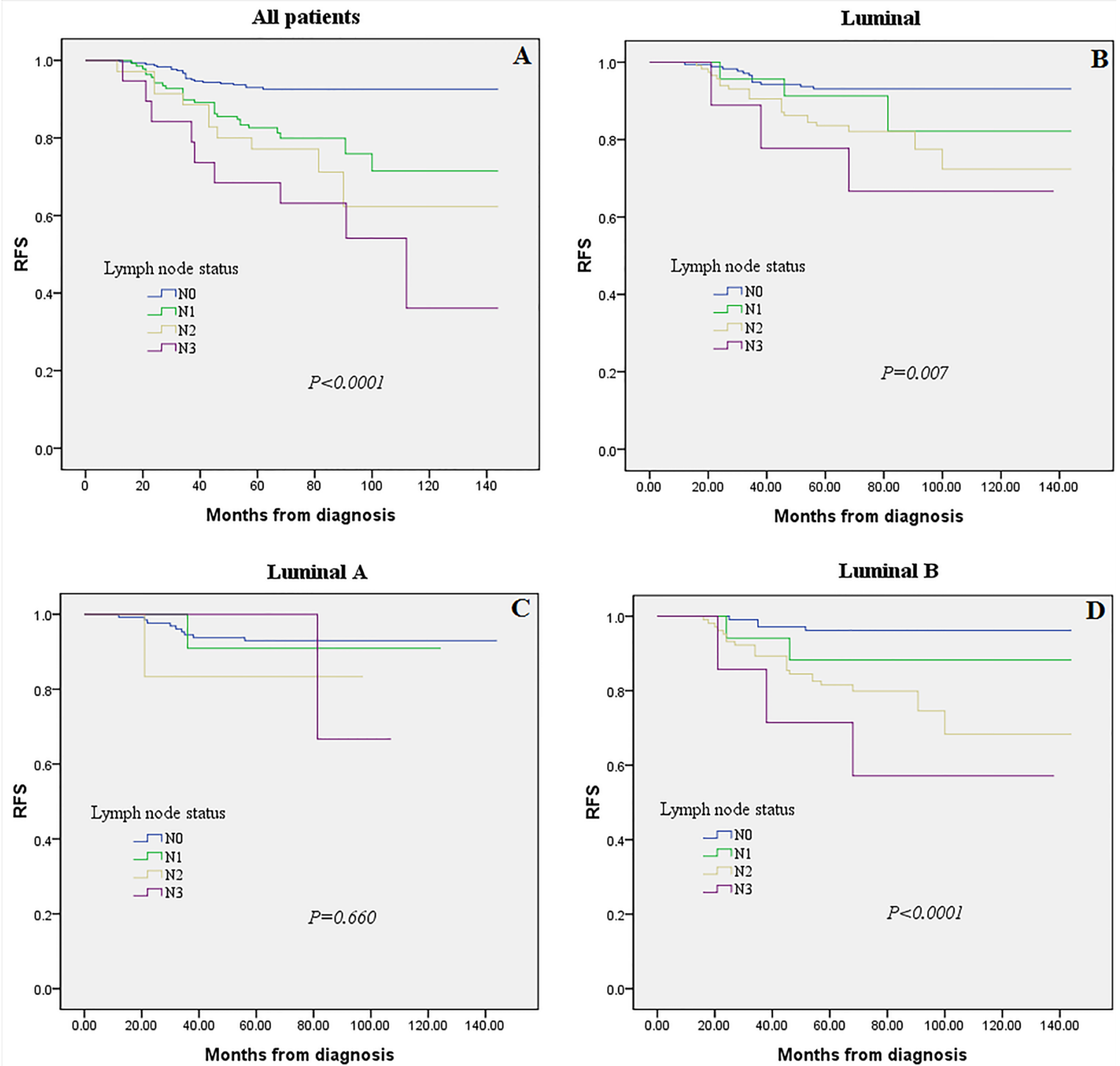

Her-2 positive

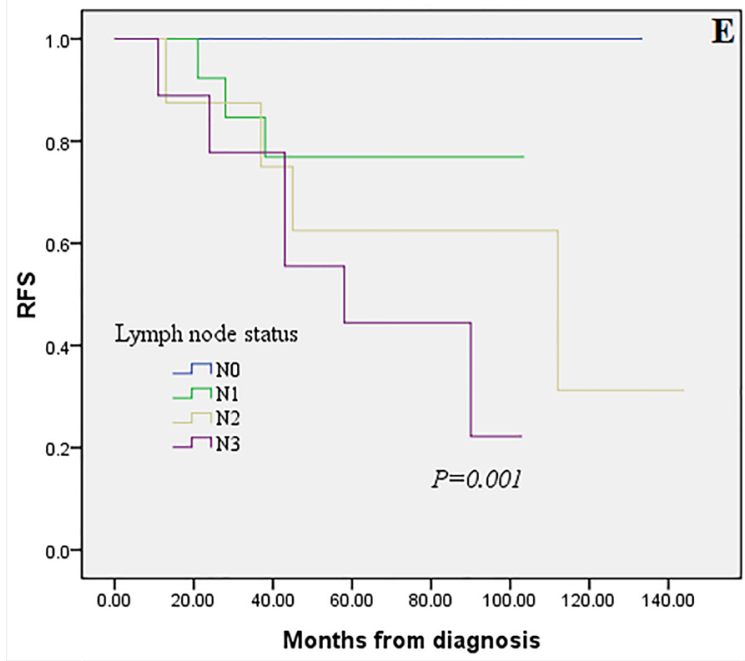

Triple negative

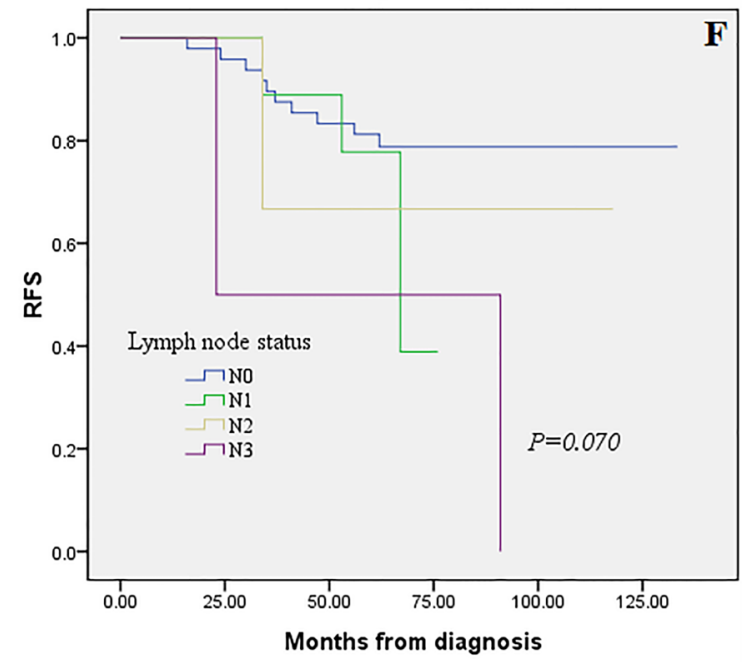

Figure 2: Kaplan-Meier analysis for recurrence-free survival according to lymph node (LN) status were stratified into four groups (N0, N1, N2 and N3). Survival analysis was performed in all 528 patients with follow-up and LNs information A., among Luminal tumors B., or in the subgroups of Luminal A tumors C., Luminal B tumors D., Her-2 positive E., and Triple negative subtype F. 

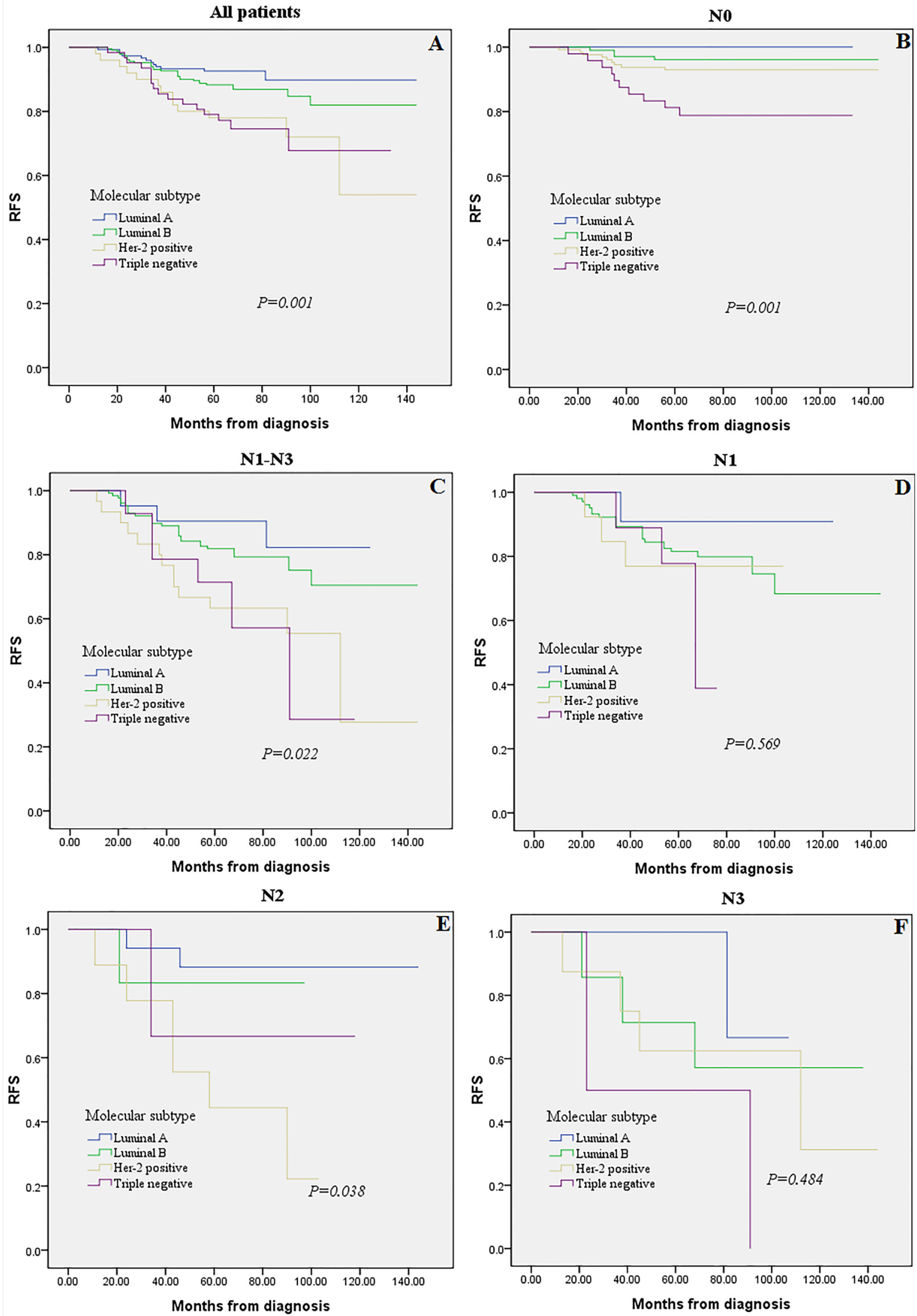

Figure 3: Kaplan-Meier analysis for recurrence-free survival according to molecular subtype. Survival analysis was performed in all 528 patients A., LN negative(N0) tumors B., LN positive(N1-N3) tumors C., or in the subgroups of N1 tumors D., N2 tumors E., and N3 tumors $\mathbf{F}$. 
suggesting that Triple negative breast cancer has nodal involvement less frequently (Table 2).

\section{The prognostic impact of $L N$ status in different molecular subtypes: Kaplan-Meier plots}

Five hundred and twenty eight patients with complete prognostic information and $\mathrm{LN}$ data were subjected to Kaplan-Meier analysis regarding the association between LN status (i.e., N0, N1, N2 and N3) and prognosis (i.e., RFS). Overall, patients showed significant differences in prognosis associated with LN status (Figure $2 \mathrm{~A}, P<0.0001$ ). When restricting the analysis to Luminal group, still a highly significant association of $\mathrm{N} 3$ with poor prognosis was retained (Figure 2B, $P=0.007$ ). While we detected no significant effect in Luminal A subgroups when analyzing Luminal A and Luminal B separately (Figure 2C, 2D, $P=0.660$ and $P<0.0001$, respectively). This effect may be casued by the worse prognosis of Luminal B compared to Luminal A cancers, since the frequency of the Luminal B subtype is nearly doubled in N1 patients ( $62.8 v s .37 .2 \%, P<0.001$; Table 1). We observed a clearly significant association between LN status among patients with Her-2 positive breast cancer (Figure 2E, $P=0.001$ ). In contrast, no significant effect of LN status was detected in the Triple negative subtype (Figure 2F, $P=0.070$ ).

\section{The prognostic impact of molecular subtypes in different LN status: Kaplan-Meier plots}

As shown in Figure 3, the association between MST (i.e., Luminal A, Luminal B, Her-2 positive, Triple negative) and prognosis (i.e., RFS) was examined. Reduced RFS rates are observed in the Her-2 positive and Triple negative subtypes (Figure $3 \mathrm{~A}, P=0.001$ ). When restricting the analysis to $\mathrm{LN}$ negative(N0) and LN positive (N1-N3) group, still a highly significant association of Triple negative breast cancer with poor prognosis was retained (Figure 3B, 3C, $P=0.001, P=$ 0.022 , respectively). While we detected no significant effect in N1 and N3 subgroups among different MST when analyzing $\mathrm{LN}$ positive group separately (Figure $3 \mathrm{D}, 3 \mathrm{~F}, P$ $=0.569$ and $P=0.484$, respectively).

\section{The clinicolpathology characteristics and survival: proportional hazards model}

We next studied whether the prognostic value that we had observed remains statistically significant in a multivariate analysis in the total cohort. We applied a multivariate Cox regression model which includes lymph node status, molecular subtype of the tumor, tumor size, tumor grade, and lymphovascular invasion. As presented in Table 3, all three, namely, LN status $(P<0.01)$, MST $(P$ $<0.01)$, and tumor size $(P<0.01)$ were significantly and independently prognostic factors in this model.

To predict the survival of patients, prognostic nomogram was depicted by Cox regression model analysis using all the significant independent indicators for recurrence (Figure 4). The nomogram can predict the probability of recurrence patients within 3 or 5 years. The c-index of the nomogram for recurrence prediction was 0.70 .

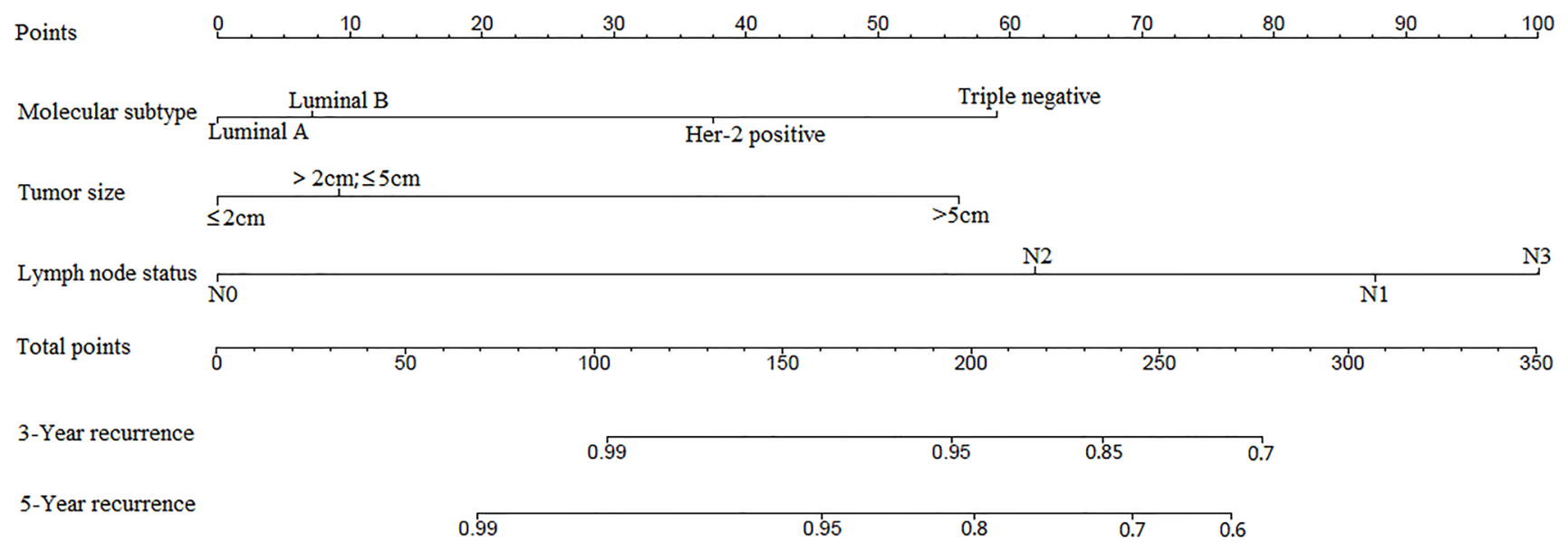

Figure 4: Postoperative nomogram with significant clinicopathologic characteristics predicted the probability of recurrence. To use the nomogram, the value attributed to an individual patient is located on each variable axis, and a line is drawn upward to determine the number of points received for each variable value. The sum of these numbers is located on the total points axis, and a line is then drawn downward to the survival axis to determine the 3-year and 5-year LRR likelihood. 


\section{DISCUSSION}

Breast cancer is a complex, heterogeneous disease at the molecular level. Gene expression studies have identified molecular subtypes (MST) with distinct clinical characteristics in different patients even in different ethnic populations [21]. The immunohistochemistry (IHC) classification of patients has been shown to correlate well with intrinsic classification using gene expression microarrays: ER/PR+, Her-2- with Luminal A; ER/ PR+, Her-2+ with Luminal B; ER-, PR-, Her-2+ (Her-2 positive); and ER-/PR-, Her-2- with Triple negative tumors $[22,23]$. Triple negative breast cancer has emerged as a group of breast cancer patients with unique therapeutic challenges and worst outcomes, and forms an important area of research interest [24]. Lymph node (LN) status is one of the most robust factors correlated to overall survival in breast cancer patients, and as such, it has been a major determinant in therapeutic decision making [25]. In the present study, we used a cohort of samples with gene expression data obtained from 528 patients with breast cancer to study influence of MST and LN status on prognosis. Our study confirmed the lower risk of axillary lymph nodal (ALN) involvement in Triple negative breast cancer patients. An decreased frequency of LN metastasis were found in Triple negative breast cancer and Luminal A patients (Table 1 and Figure 1). This was not unexpected and in line with several earlier reports [26-28].

Our study demonstrated tumor size as a significant independent predictive factor for positive LN status with an odds ratio of 1.62 for T3 $(>5 \mathrm{~cm})$ versus $\mathrm{T} 1(\leq 2 \mathrm{~cm})$ tumors. The results are in accordance with Viale et al [2] and Silverstein et al [29] analysis, they demonstrated that tumor size to be the most significant predictor of LN metastases. In addition, older patients ( $>50$ years old) were $50 \%$ more likely to have positive LNs. With respect to lymphovascular invasion (LVI), a variety of studies have shown strong correlation with ALN involvement. In this study, LVI was a predictor of LN positivity with an odds ratio of 1.47, but the Triple negative phenotype was not associated with LN positivity. However, we found that $\mathrm{LN}$ involvement is more frequently observed in Her-2+ tumors (Luminal B and Her-2 positive) as compared to Luminal A and Triple negative subtypes.

Our study has several limitations. Firstly, this study is a retrospective analysis and the number of samples is relatively few. Thus, differences in treatment depending on LN status and molecular subtype at diagnosis cannot be excluded and have the potential to significantly introduce bias into our analyses. A prospective multicentre randomised clinical study should be performed in the future and the results will provide more scientific basis and reliable guidance for judgement of clinical treatment. Second, most patients did not undergo trastuzumab treatment in Her-2 positive patients which has been shown to decrease rates of LRR by $50 \%$ [30]. The reason for lack of adjuvant trastuzumab is that during the period of study enrollment (January 2004 to December 2009), the concept of adjuvant trastuzumab had not been built up completely.

Previously studies suggested that the aggressive nature of Triple negative breast cancer may be due to distant spread in disease early stage $[3,31,32]$. Dent et al [33] showed that patients with Triple negative breast cancer had a greater risk of distant recurrence by means of visceral metastases. Together with the results of our study, it could be hypothesized Triple negative breast cancer has less lymph node metastasis but is more aggressively. It may due to hematogenous spread or lack of targetable treatment. If Triple negative breast cancer is more likely to spread hematogenously, adjuvant systemic treatment might be more beneficial than locoregional radiotherapy and axillary treatment in patients with early TNBC. This requires further investigation to study why triple negative breast cancer have less lymph node metastasis in a serious of clinical and basic research.

In conclusion, we demonstrated that association of LN status with breast cancer MST contributes to its important role as prognostic factor among patients with breast cancer. On the other hand, although Triple negative breast cancer is more aggressive, it does not metastasize more frequently to the axilla and it is not associated more frequently with a higher number of involved nodes. This may indicate that Triple negative breast cancer tends to spread hematogenously rather than lymphogenously, stressing the importance of systemic treatment compared to locoregional treatment in these patients.

\section{Ethical approval}

This study was approved by the Bao Di Clinical College of Tianjin Medical University and Tianjin Medical University Cancer Institute and Hospital, China and has been performed in accordance with the ethical standards laid down in the 1964 Helsinki declaration and its later amendments.

\section{Synopsis}

Our study analyse and compare survival outcomes of breast cancer patients between different molecular subtype and lymph nodes status. Data showed that Triple negative and Luminal A breast cancers were more frequently node-negative. On the other hand, we demonstrated that the nomogram can predict the probability of recurrence patients within 3 or 5 years.

\section{ACKNOWLEDGMENTS}

This work was financially supported by Tianjin municipal health bureau of science and technology fund (2010KZ02). 


\section{CONFLICTS OF INTEREST}

There is no conflict of interest.

\section{REFERENCES}

1. Yoshihara E, Smeets A, Laenen A, Reynders A, Soens J, Van Ongeval C, Moerman P, Paridaens R, Wildiers H, Neven P, Christiaens MR. Predictors of axillary lymph node metastases in early breast cancer and their applicability in clinical practice. Breast. 2013; 22:357-61. https://doi. org/10.1016/j.breast.2012.09.003

2. Viale G, Zurrida S, Maiorano E, Mazzarol G, Pruneri G, Paganelli G, Maisonneuve P, Veronesi U. Predicting the status of axillary sentinel lymph nodes in 4351 patients with invasive breast carcinoma treated in a single institution. Cancer. 2005; 103:492-500. https://doi.org/10.1002/ cncr.20809

3. Crabb SJ, Cheang MC, Leung S, Immonen T, Nielsen TO, Huntsman DD, Bajdik CD, Chia SK. Basal breast cancer molecular subtype predicts for lower incidence of axillary lymph node metastases in primary breast cancer. Clin Breast Cancer. 2008; 8:249-56. https://doi.org/10.3816/ CBC.2008.n.028

4. Perou CM, Sørlie T, Eisen MB, van de Rijn M, Jeffrey SS, Rees CA, Pollack JR, Ross DT, Johnsen H, Akslen LA, Fluge O, Pergamenschikov A, Williams C, et al. Molecular portraits of human breast tumours. Nature. 2000; 406:74752. https://doi.org/10.1038/35021093

5. Wiechmann L, Sampson M, Stempel M, Jacks LM, Patil SM, King T, Morrow M. Presenting features of breast cancer differ by molecular subtype. Ann Surg Oncol. 2009; 16:2705-10. https://doi.org/10.1245/s10434-009-0606-2

6. Bevilacqua JL, Kattan MW, Fey JV, Cody HS 3rd, Borgen PI, Van Zee KJ. Doctor, what are my chances of having a positive sentinel node? A validated nomogram for risk estimation. J Clin Oncol. 2007; 25:3670-79. https://doi. org/10.1200/JCO.2006.08.8013

7. Hwang RF, Krishnamurthy S, Hunt KK, Mirza N, Ames FC, Feig B, Kuerer HM, Singletary SE, Babiera G, Meric F, Akins JS, Neely J, Ross MI. Clinicopathologic factors predicting involvement of nonsentinel axillary nodes in women with breast cancer. Ann Surg Oncol. 2003; 10:248 54. https://doi.org/10.1245/ASO.2003.05.020

8. Van Zee KJ, Kattan MW. Validating a predictive model for presence of additional disease in the non-sentinel lymph nodes of a woman with sentinel node positive breast cancer. Ann Surg Oncol. 2007; 14:2177-78. https://doi. org/10.1245/s10434-007-9361-4

9. Banz-Jansen C, Heinrichs A, Hedderich M, Waldmann A, Wedel B, Mebes I, Diedrich K, Rody A, Fischer D. Are there changes in characteristics and therapy of young patients with early-onset breast cancer in Germany over the last decade? Arch Gynecol Obstet. 2013; 288:379-83. https://doi.org/10.1007/s00404-013-2738-7
10. Gluz O, Liedtke C, Gottschalk N, Pusztai L, Nitz U, Harbeck N. Triple-negative breast cancer-current status and future directions. Ann Oncol. 2009; 20:1913-27. https://doi.org/10.1093/annonc/mdp492

11. Liedtke C, Mazouni C, Hess KR, André F, Tordai A, Mejia JA, Symmans WF, Gonzalez-Angulo AM, Hennessy B, Green M, Cristofanilli M, Hortobagyi GN, Pusztai L. Response to neoadjuvant therapy and long-term survival in patients with triple-negative breast cancer. J Clin Oncol. 2008; 26:1275-81. https://doi.org/10.1200/ JCO.2007.14.4147

12. Arvold ND, Taghian AG, Niemierko A, Abi Raad RF, Sreedhara M, Nguyen PL, Bellon JR, Wong JS, Smith BL, Harris JR. Age, breast cancer subtype approximation, and local recurrence after breast-conserving therapy. J Clin Oncol. 2011; 29:3885-91. https://doi.org/10.1200/ JCO.2011.36.1105

13. Reyal F, Rouzier R, Depont-Hazelzet B, Bollet MA, Pierga JY, Alran S, Salmon RJ, Fourchotte V, Vincent-Salomon A, Sastre-Garau X, Antoine M, Uzan S, Sigal-Zafrani B, De Rycke Y. The molecular subtype classification is a determinant of sentinel node positivity in early breast carcinoma. PLoS One. 2011; 6:e20297. https://doi. org/10.1371/journal.pone.0020297

14. Freedman GM, Anderson PR, Li T, Nicolaou N. Locoregional recurrence of triple-negative breast cancer after breast-conserving surgery and radiation. Cancer. 2009; 115:946-51. https://doi.org/10.1002/cncr.24094

15. Holm-Rasmussen EV, Jensen MB, Balslev E, Kroman N, Tvedskov TF. Reduced risk of axillary lymphatic spread in triple-negative breast cancer. Breast Cancer Res Treat. 2015; 149:229-36. https://doi.org/10.1007/s10549-0143225-y

16. Hammond ME, Hayes DF, Dowsett M, Allred DC, Hagerty KL, Badve S, Fitzgibbons PL, Francis G, Goldstein NS, Hayes M, Hicks DG, Lester S, Love R, et al. American Society of Clinical Oncology/College Of American Pathologists guideline recommendations for immunohistochemical testing of estrogen and progesterone receptors in breast cancer. J Clin Oncol. 2010; 28:2784-95. https://doi.org/10.1200/JCO.2009.25.6529

17. Bianchi S, Caini S, Paglierani M, Saieva C, Vezzosi V, Baroni G, Simoni A, Palli D, Tuscany Breast Cancer Study Group. Accuracy and reproducibility of HER2 status in breast cancer using immunohistochemistry: A quality control study in Tuscany evaluating the impact of updated 2013 ASCO/CAP recommendations. Pathol Oncol Res. 2015; 21:477-85. https://doi.org/10.1007/s12253-0149852-0

18. Untch M, Gerber B, Harbeck N, Jackisch C, Marschner N, Möbus V, von Minckwitz G, Loibl S, Beckmann MW, Blohmer JU, Costa SD, Decker T, Diel I, et al. 13th st. Gallen international breast cancer conference 2013: primary therapy of early breast cancer evidence, controversies, consensus - opinion of a german team of experts (zurich 
2013). Breast Care (Basel). 2013; 8:221-29.

19. Kobayashi T, Iwaya K, Moriya T, Yamasaki T, Tsuda H, Yamamoto J, Matsubara O. A simple immunohistochemical panel comprising 2 conventional markers, Ki67 and p53, is a powerful tool for predicting patient outcome in luminaltype breast cancer. BMC Clin Pathol. 2013; 13:5. https:// doi.org/10.1186/1472-6890-13-5

20. Yang Y, Zhang YJ, Zhu Y, Cao JZ, Yuan ZY, Xu LM, Wu JX, Wang W, Wu T, Lu B, Zhu SY, Qian LT, Zhang FQ, et al. Prognostic nomogram for overall survival in previously untreated patients with extranodal NK/T-cell lymphoma, nasal-type: a multicenter study. Leukemia. 2015; 29:157177. https://doi.org/10.1038/leu.2015.44

21. Jagsi R, Pierce L. Postmastectomy radiation therapy for patients with locally advanced breast cancer. Semin Radiat Oncol. 2009; 19:236-43. https://doi.org/10.1016/j. semradonc.2009.05.009

22. Carey LA, Dees EC, Sawyer L, Gatti L, Moore DT, Collichio F, Ollila DW, Sartor CI, Graham ML, Perou CM. The triple negative paradox: primary tumor chemosensitivity of breast cancer subtypes. Clin Cancer Res. 2007; 13:2329-34. https://doi.org/10.1158/1078-0432. CCR-06-1109

23. Carey LA, Perou CM, Livasy CA, Dressler LG, Cowan D, Conway K, Karaca G, Troester MA, Tse CK, Edmiston S, Deming SL, Geradts J, Cheang MC, et al. Race, breast cancer subtypes, and survival in the Carolina Breast Cancer Study. JAMA. 2006; 295:2492-502. https://doi. org/10.1001/jama.295.21.2492

24. Agarwal G, Nanda G, Lal P, Mishra A, Agarwal A, Agrawal V, Krishnani N. Outcomes of Triple-Negative Breast Cancers (TNBC) Compared with Non-TNBC: Does the Survival Vary for All Stages? World J Surg. 2016; 40:1362-72. https://doi.org/10.1007/s00268-016-3422-4

25. Gangi A, Mirocha J, Leong T, Giuliano AE. Triple-negative breast cancer is not associated with increased likelihood of nodal metastases. Ann Surg Oncol. 2014; 21:4098-103. https://doi.org/10.1245/s10434-014-3989-7

26. de Kruijf EM, Bastiaannet E, Rubertá F, de Craen AJ, Kuppen PJ, Smit VT, van de Velde CJ, Liefers GJ. Comparison of frequencies and prognostic effect of molecular subtypes between young and elderly breast cancer patients. Mol Oncol. 2014; 8:1014-25. https://doi. org/10.1016/j.molonc.2014.03.022
27. Jenkins EO, Deal AM, Anders CK, Prat A, Perou CM, Carey LA, Muss HB. Age-specific changes in intrinsic breast cancer subtypes: a focus on older women. Oncologist. 2014; 19:1076-83. https://doi.org/10.1634/ theoncologist.2014-0184

28. Azim HA Jr, Michiels S, Bedard PL, Singhal SK, Criscitiello C, Ignatiadis M, Haibe-Kains B, Piccart MJ, Sotiriou C, Loi S. Elucidating prognosis and biology of breast cancer arising in young women using gene expression profiling. Clin Cancer Res. 2012; 18:1341-51. https://doi.org/10.1158/1078-0432.CCR-11-2599

29. Silverstein MJ, Skinner KA, Lomis TJ. Predicting axillary nodal positivity in 2282 patients with breast carcinoma. World J Surg. 2001; 25:767-72. https://doi.org/10.1007/ s00268-001-0003-x

30. Romond EH, Perez EA, Bryant J, Suman VJ, Geyer CE Jr, Davidson NE, Tan-Chiu E, Martino S, Paik S, Kaufman PA, Swain SM, Pisansky TM, Fehrenbacher L, et al. Trastuzumab plus adjuvant chemotherapy for operable HER2-positive breast cancer. N Engl J Med. 2005; 353:1673-84. https://doi.org/10.1056/NEJMoa052122

31. Dent R, Trudeau M, Pritchard KI, Hanna WM, Kahn HK, Sawka CA, Lickley LA, Rawlinson E, Sun P, Narod SA. Triple-negative breast cancer: clinical features and patterns of recurrence. Clin Cancer Res. 2007; 13:4429-34. https:// doi.org/10.1158/1078-0432.CCR-06-3045

32. Gann PH, Colilla SA, Gapstur SM, Winchester DJ, Winchester DP. Factors associated with axillary lymph node metastasis from breast carcinoma: descriptive and predictive analyses. Cancer. 1999; 86:1511-19. https://doi. org/10.1002/(SICI)1097-0142(19991015)86:8<1511::AIDCNCR18>3.0.CO;2-D

33. Dent R, Hanna WM, Trudeau M, Rawlinson E, Sun P, Narod SA. Pattern of metastatic spread in triple-negative breast cancer. Breast Cancer Res Treat. 2009; 115:423-28. https://doi.org/10.1007/s10549-008-0086-2. 